\title{
Cannabis medicinal como recurso terapéutico: estudio preliminar
}

Julia Galzerano Guida*, Cecilia Carina Orellana Navone ${ }^{\dagger}$, María Daniela Ríos Pérez, Ana Laura Coitiño González§, Pablo Mariano Velázquez Ramos"

\section{Resumen}

Introducción: los productos de Cannabis sativa L poseen una eficacia terapéutica conocida desde la Antigüedad, aunque su tipificación farmacológica data de mediados del siglo XX. Recientemente se regularizó su uso en Uruguay.

Objetivo: analizar una experiencia clínico terapéutica preliminar con cannabis medicinal (CM) con alto contenido en cannabidiol (CBD).

Método: estudio epidemiológico observacional y retrospectivo de una cohorte de 355 pacientes que concurrieron a consultar espontáneamente sobre CM en una clínica privada entre agosto de 2016 y diciembre de 2017. Durante la primera entrevista se recogieron datos demográficos, historia clínica, farmacológica, expectativas y experiencia previa con cannabis. Se indicó mayoritariamente cannabis con alto contenido en $\operatorname{CBD}(5,25 \%$ de CBD y $0,2 \%$ de tetrahidrocannabinol [THC]). En consultas siguientes, se investigó el acceso al CM y se valoró su respuesta y efectos adversos mediante escalas analógicas. Se utilizó estadística descriptiva.

Resultados: en la cohorte estudiada predominaron mujeres con edad promedio de 67 años, de nivel educativo terciario. Las patologías de consulta fueron neurológicas (38\%), enfermedades reumáticas o artro-degenerativas (37\%), neoplasias $(13 \%)$, psiquiátricas (4\%) y misceláneas (8\%). La mayoría de estos casos $(60,6 \%)$ refirió mejoría de sus síntomas y solo 16,3\% de la población estudiada presentó efectos adversos de grado leve. Los altos costos y la gestión dificultosa para conseguir el CM fueron causas para no iniciar o abandonar el tratamiento.

Conclusiones: nuestro estudio preliminar refleja una respuesta terapéutica positiva y sin efectos adversos significativos al CM con alto contenido de CBD. El 60,6\% de los pacientes tratados refirió mejoría de sus síntomas. Los factores decisivos para un tratamiento exitoso orientan a facilitar el acceso al CM, mejorando la gestión para su obtención y disminuyendo los costos para una mayor accesibilidad.

Palabras clave: Cannabis

Marihuana medicinal

Key words: $\quad$ Cannabis

Plantas medicinales

Medical marijuana

Cannabidiol

Plants, medicinals

Aceites vegetales

Cannabidiol

Cannabinoides

Plant oils

Cannabinoides

\footnotetext{
* Médico internista, especialista en VIH y adicciones. Coordinadora de adicciones del CASMU- IAMPP. Integrante Policlínica VIH CASMU-IAMPP.

† Neuróloga y neurofisióloga clínica. Servicio de Neurología del Hospital Maciel y Departamento de Neurofisiología del Hospital Británico. ‡ Ex residente de Medicina Familiar y Comunitaria (ASSE). Docente asociada a la Cátedra de Medicina Familiar y Comunitaria. $\S$ Ex residente de Medicina Familiar y Comunitaria (CASMU).

Tा Médico general ASSE SAI-PPL. Medicina tradicional china.

Correspondencia: Dra. Julia Galzerano. Etna 5870, Montevideo, Uruguay. Correo electrónico: juliagalzerano@gmail.com

Los participantes de este trabajo declaran tener un consultorio privado de medicina cannábica.

Recibido: 29/5/19

Aprobado: 25/9/19
} 


\section{Introducción}

En la actualidad se conoce que Cannabis sativa L es una especie herbácea del género cannabaceae que ha sido utilizada desde la Antigüedad como alimento, fuente de fibra, droga y medicina ${ }^{(1,2)}$. Recientemente su empleo terapéutico en pacientes con patologías crónicas de difícil tratamiento es objeto de estudios clínicos, además de usarse frecuentemente off label en búsqueda de soluciones terapéuticas que contribuyan a mejorar síntomas discapacitantes y la calidad de vida. En los últimos tiempos se han efectuado y continúan desarrollándose estudios científicos acerca de la fisiología del sistema endocannabinoide, lo que justificaría la potencial utilización terapéutica del cannabis como interesante recurso en el ámbito de la salud ${ }^{(3,4)}$. Este aspecto resulta todavía controversial en los espacios de la medicina, ya que el cannabis ha sido estigmatizado por su uso recreativo, razón por la cual no fue estudiado ni incorporado en forma analítica y crítica como fármaco de utilidad en diversas situaciones clínicas.

El sistema endocannabinoide humano, encargado de modular múltiples procesos orgánicos específicos, posee ligandos endógenos y enzimas de producción pro$\mathrm{pia}^{(5)}$. Los receptores más importantes son el receptor cannabinoide tipo 1 (RCB1) y receptor cannabinoide tipo 2 (RCB2), a los cuales pueden unirse tanto ligandos endógenos: anandamida y el 2-araquidonilglicerol (2AG), como también fitocannabinoides: delta-9-THC (tetrahidrocannabinol) y cannabidiol (CBD) ${ }^{(4,5)}$. El sistema nervioso central y el periférico son los sitios principales de expresión de RCB1, hallándose en menor proporción en otros tejidos orgánicos. Los RCB2 predominan en el sistema inmune y en menor cantidad en tejidos gonadales y áreas restringidas del cerebro ${ }^{(6)}$.

Diversos países, a partir de hallazgos científicos, están revisando las medidas restrictivas y aprobando el empleo de CM como parte del arsenal terapéutico ${ }^{(7)}$. En Uruguay, el proceso de regulación del uso del cannabis se inicia en el año 2012 cuando se comienza a hablar del control estatal ${ }^{(8)}$, continuando en 2013 con la aprobación de la Ley 19172 (cultivo doméstico, clubes de cannabis, venta de flores de cannabis en farmacias y $\mathrm{CM})^{(9)}$. El uso de CM se reglamentó en $2015^{(10)}$ y al año tuvo lugar en nuestro país el Primer Curso de Medicina Cannábica de Latinoamérica, que organizó el Sindicato Médico del Uruguay (SMU) y fue cocoordinado por el SMU y el International Center Ethnobotanical Education Research (ICEERS). Contó con el apoyo de la Junta Nacional de Drogas, el Instituto de Regulación y Control del Cannabis (IRCCA) y el auspicio de otras instituciones, por ejemplo, la Escuela de Graduados. En octubre de 2017 se autorizó la venta en farmacias de aceite de can- nabis elaborado en base a extractos de variedades no psicoactivas $^{(11)}$.

Desde el año 2016 funciona en Montevideo una clínica para el tratamiento con CM de carácter privado, integrada por distintos profesionales de la salud y que constituye el primer espacio de atención interdisciplinaria en la materia en nuestro país. Ha sido interés de quienes forman parte de este equipo, dado el carácter innovador de esta experiencia, efectuar una revisión crítica del trabajo realizado. A tales efectos fue propuesto un estudio observacional retrospectivo que diera cuenta de diversos aspectos referidos a las características de la población asistida y los resultados terapéuticos obtenidos en un período de tiempo establecido.

\section{Objetivos}

\section{Objetivo general}

Describir las características clínicas y los resultados terapéuticos encontrados con el uso de $\mathrm{CM}$ en una cohorte de pacientes que consultaron en la clínica entre agosto de 2016 y diciembre de 2017.

\section{Objetivos específicos}

1. Determinar las características sociodemográficas de la población que concurrió a la clínica para acceder a un tratamiento con $\mathrm{CM}$ en el período mencionado.

2. Evaluar la respuesta terapéutica al CM con alto contenido en CBD en diferentes patologías.

3. Registrar la presencia de efectos adversos en la población analizada.

\section{Material y método}

\section{Diseño del estudio}

Se planteó un diseño epidemiológico de tipo observacional y retrospectivo de una cohorte de pacientes que concurrieron a consulta por CM entre agosto de 2016 y diciembre de 2017 y que cumplían con los criterios de inclusión explicitados más adelante.

Las variantes estudiadas fueron: variable cuantitativa continua: edad; variable cualitativa nominal: sexo; variables cualitativas categóricas: escolaridad, cobertura de salud, expectativa frente al uso de cannabis y efectividad. Estas últimas se midieron con la escala ordinal de Likert.

\section{Población estudiada y criterios de selección}

Los criterios de inclusión fueron: pacientes mayores de 18 años que habiendo recibido los tratamientos estándares habituales para sus patologías no presentaron buena respuesta terapéutica; los criterios de exclusión fueron ser menores de 18 años y tener contraindicaciones para 
el uso de cannabis, como cardiopatía isquémica descompensada, arritmia no controlada o psicosis descompensada.

Por tratamiento estándar habitual se entienden aquellos tratamientos que son reconocidos y se utilizan en la clínica de forma permanente como recursos terapéuticos de elección y científicamente avalados frente a las distintas patologías existentes. En el caso de este estudio no se valoró la necesidad de incorporar el arsenal terapéutico recibido por los 355 pacientes que consultaron, ya que excedía con creces los alcances de este trabajo.

En el período comprendido entre agosto de 2016 y diciembre de 2017 consultaron 355 pacientes en una clínica privada para tratamiento con $\mathrm{CM}$, los cuales fueron asesorados y se les completó los formularios a fin de iniciar los trámites para la importación de aceite de cannabis.

\section{Modalidad de trabajo}

Todos los pacientes concurrieron a una o más consultas. Recibieron información sobre el sistema endocannabinoide mediante video explicativo previo a la primera consulta. Se les asesoró sobre los efectos terapéuticos del cannabis, con especial énfasis en proyectar expectativas realistas de acuerdo a los conocimientos científicos más actualizados. Se explicaron las diferencias entre el CM artesanal y el CM regulado, costos y trámites, tiempos de estas gestiones, interacciones farmacológicas y posibles efectos adversos. Se les solicitó autorización para un relevamiento anónimo de los datos con la posibilidad de incorporarlos a un estudio posterior.

Durante la primera consulta se realizó una entrevista semiestructurada que incluía: a) datos patronímicos (edad, sexo, escolaridad, cobertura de salud); b) patologías que motivaron la consulta, datos evolutivos de éstas, tratamiento previo; c) antecedentes patológicos personales, evaluando contraindicaciones; d) expectativas del paciente frente al uso de $\mathrm{CM}$; e) experiencia previa, ya fuese uso recreativo o medicinal, así como la respuesta a la misma, y f) examen físico pertinente a cada caso clínico. La evaluación se realizó en la segunda, tercera y sexta consultas, en un período aproximado de siete meses.

A quienes tenían indicación de tratamiento, se les prescribió CM con alto contenido de $\mathrm{CBD}$ en forma de aceites, en proporciones de $5 \%$ a 7,5\%, acompañado de dosis bajas de THC $(0,2 \%$ - 0,9\%). Estos aceites cuentan con la aprobación de importación del Ministerio de Salud y poseen determinación cromatográfica de la concentración de cada cannabinoide realizada en origen. $\mathrm{Su}$ producción cumple con las normas de buenas prácticas de manufactura (GMP) que certifican la ausencia de metales pesados, bacterias, hongos u otros contaminantes tóxicos. El aceite de cannabis que se comercializa en Uruguay (CBD: $2 \%$ - 5\%, THC: 0,2\%) no se encontraba aún disponible, ya que comenzó su venta en farmacias a partir de diciembre de 2017. Los pacientes utilizaron la vía sublingual en dosis diarias progresivamente crecientes de acuerdo a respuesta y tolerancia. Se les exhortó a continuar con la medicación que recibían y evitar reducciones de dosis o suspensiones hasta consultar a su médico tratante.

Las consultas de control evolutivo incluyeron: 1) acceso al producto y dificultades encontradas; 2) beneficio observado en el alivio de síntomas reportados y grado de dicha respuesta valorada por escala ordinal tipo Likert de tres puntos: a) sin cambios; b) mejoría leve a moderada; c) mejoría importante; 3) evaluación de efectos adversos y gravedad de éstos de acuerdo a pautas de CTCAE v5.0 $0^{(12,13)}$.

\section{Estadística}

Se utilizaron métodos de estadística descriptiva de datos cuantitativos discretos y cualitativos, con intervalos de clase, medidas de resumen y cálculo de las frecuencias absolutas y relativas de las variables estudiadas.

\section{Normas éticas}

El estudio fue realizado respetando las recomendaciones internacionales sobre investigación clínica (Declaración de Helsinki de la Asociación Médica Mundial de 1975 y sus posteriores enmiendas) ${ }^{(14)}$ y la Declaración de Taipei, complementaria de la de Helsinski y referida a investigación sobre bases de datos, datos masivos y biobancos, particularmente en la declaración de principios de los parágrafos $1,2,3,5,8,9,10$ y $11^{(15)}$.

Se solicitó a los pacientes autorización para un relevamiento anónimo de los datos, con la posibilidad de incorporarlos para el desarrollo de estudios posteriores acerca de los beneficios observados por el uso terapéutico del cannabis.

\section{Resultados}

\section{Consideraciones generales}

El comportamiento del total de los 355 pacientes que acudieron a la consulta se distribuyó del siguiente modo: 259 (73\%) continuaron el tratamiento hasta el final del estudio; 46 pacientes $(12,9 \%)$ concurrieron a una sola consulta; 35 (9,9\%) plantearon que acudieron solamente en busca de asesoramiento. Los 15 pacientes restantes $(4,2 \%)$ suspendieron su continuidad por varios motivos: en nueve casos se consideró inviable el tratamiento a causa de los elevados costos o problemas para completar las gestiones requeridas para la importación; en un paciente se produjo suspensión por efecto 
Tabla 1. Datos demográficos.

\begin{tabular}{lccc}
\hline Edades & Mujeres & Hombres & Total pacientes \\
\hline $19-40$ años & 28 & 20 & $48(13,5 \%)$ \\
$41-60$ años & 67 & 31 & $98(27,6 \%)$ \\
$61-100$ años & 141 & 68 & $209(58,9 \%)$ \\
Totales & $236(66,5 \%)$ & $119(33,5 \%)$ & $355(100 \%)$ \\
\hline
\end{tabular}

Tabla 3. Expectativas personales.

\begin{tabular}{lc}
\hline Síntomas principales & Número de pacientes \\
\hline Dolor & $185(52,0 \%)$ \\
Síntomas neurológicos & $96(27,1 \%)$ \\
Síntomas psiquiátricos & $21(5,9 \%)$ \\
Trastorno del sueño & $3(0,8 \%)$ \\
Alteración cutánea & $2(0,6 \%)$ \\
Síndrome de Cushing & $1(0,3 \%)$ \\
Tinnitus & $2(0,6 \%)$ \\
Otros síntomas & $45(12,7 \%)$ \\
Total & $355(100 \%)$ \\
\hline
\end{tabular}

adverso (rash peribucal) y cinco casos que presentaban patologías neoplásicas terminales fallecieron durante el estudio.

\section{Caracterización demográfica}

Estos datos se resumen en la tabla 1. Se incluyeron 355 pacientes protocolizados durante el período establecido, con edad media de 60 años (19-94). Se destaca la prevalencia de pacientes mayores a 60 años $(58,9 \%)$, el sexo femenino $(66,5 \%)$ y la pertenencia al sistema de salud privado (90\%). La experiencia previa con cannabis, ya fuese medicinal o recreativo, fue registrada en $23 \%$ de los casos. La cohorte estudiada poseía una alta tasa de escolarización con predominio de educación terciaria (tabla 2). La principal expectativa frente al uso de $\mathrm{CM}$ fue la mejora de la calidad de vida en sentido amplio y mantener objetivos personales, sociales y laborales (tabla 3).

1. Motivos de consulta: mejorar el dolor crónico refractario a la terapéutica convencional fue la consulta más frecuente $(52,1 \%)$ en esta población. Las de-
Tabla 2. Nivel educativo.

\begin{tabular}{lc}
\hline Educación & Número de pacientes \\
\hline Primaria completa & $23(6,5 \%)$ \\
Secundaria completa & $154(43,4 \%)$ \\
Terciaria & $162(45,6 \%)$ \\
Educación especial & $16(4,5 \%)$ \\
Total & $355(100 \%)$ \\
\hline
\end{tabular}

Tabla 4. Motivos de consulta principales.

\begin{tabular}{lc}
\hline Datos etiológicos & Número de pacientes \\
\hline Patologías neurológicas & $135(38 \%)$ \\
Dolor plurietiológico & $132(37 \%)$ \\
Patologías neoplásicas & $46(13 \%)$ \\
Patologías psiquiátricas & $14(4 \%)$ \\
Otras patologías & $28(8 \%)$ \\
Total & $355(100 \%)$ \\
\hline
\end{tabular}

más manifestaciones sintomáticas correspondieron principalmente a la esfera neurológico psiquiátrica: crisis convulsivas, rigidez, temblor, depresión, síntomas cognitivos, trastornos comportamentales, trastornos del sueño y espasticidad (tabla 4).

2. Diagnósticos clínicos: se resumen en la tabla 5: a) Patologías neurológicas: 135 pacientes (38\%), de las cuales las más frecuentes fueron: enfermedad de Parkinson, epilepsia, esclerosis múltiple, dolor neuropático y tumores cerebrales; b) Patologías que cursan con dolor crónico: 132 pacientes (37\% del total), siendo las más frecuentes artrosis, fibromialgia y artritis reumatoide; c) Patología neoplásica: 46 pacientes ( $13 \%$ del total): cáncer de mama, de pulmón y de colon lideran en frecuencia (los tumores cerebrales se incluyeron en enfermedades neurológicas); d) $\mathrm{Pa}$ tologías psiquiátricas: 14 pacientes ( $4 \%$ del total) en las que predominaron: depresión, trastorno bipolar y de pánico, y e) Otras patologías, 28 pacientes $(8 \%$ del total) que padecen enfermedades dermatológicas, pulmonares o gastrointestinales.

3. Elección terapéutica: se completaron los formularios para importar aceite de cannabis para 355 pacientes. A éstos se les indicó CM con alto contenido de CBD, seleccionando el más adecuado a su patología, siendo el más frecuente $5 \%$ de CBD y $0,2 \%$ de 


\begin{tabular}{|lc|}
\hline Tabla 5. Datos etiológicos. & \\
\hline Datos etiológicos & Número de pacientes \\
\hline Patologías neurológicas & $135(38 \%)$ \\
Dolor plurietiológico & $132(37 \%)$ \\
Patologías neoplásicas & $46(13 \%)$ \\
Patologías psiquiátricas & $14(4 \%)$ \\
Otras patologías & $28(8 \%)$ \\
Total & $355(100 \%)$ \\
\hline
\end{tabular}

Tabla 7. Respuesta terapéutica reportada.

\begin{tabular}{lc}
\hline Respuesta terapéutica reportada & Número de pacientes \\
\hline Mejora importante & $67(18,9 \%)$ \\
Mejora leve o moderada & $148(41,7 \%)$ \\
Sin cambios & $28(7,9 \%)$ \\
Suspenden tratamiento & $15(4,1 \%)$ \\
Solo primera consulta & $46(13 \%)$ \\
Sin tratamiento & $35(9,9 \%)$ \\
Otros & $16(4,5 \%)$ \\
Total & $355(100 \%)$ \\
\hline
\end{tabular}

THC (71\%). A $29 \%$ se les indicó preparados con $7,5 \%$ de CBD y $0,9 \%$ de THC (tabla 6). De los 355 pacientes iniciales, 46 concurrieron sólo a una consulta $(12,9 \%)$, y 309 pacientes $(87 \%)$ concurrieron a la segunda y tercera consultas realizadas uno y tres meses después del asesoramiento inicial. De estos 309, solo 274 pacientes habían comenzado el tratamiento, los restantes 35 pacientes indicaron los motivos que les impidieron tratarse: pérdida de las recetas, dificultades económicas y dificultades en la gestión de obtención. De los 274 pacientes tratados, se relevaron datos de respuesta en la tercera consulta a 259 pacientes, ya que 15 pacientes lo suspendieron: nueve lo hicieron por causas económicas e inconvenientes en la gestión de la obtención; cinco que presentaron patologías neoplásicas terminales fallecieron durante el período de estudio; un paciente suspendió por presentar un rash peribucal (figura 1). La evaluación se realizó a través de una escala ordinal de Likert de 3 puntos: a) sin cambios; b) mejoría leve a moderada; c) mejoría importante. El $61 \%$ de pacientes notaron mejoría definida de sus síntomas:

\begin{tabular}{|lc|}
\hline \multicolumn{2}{|l|}{ Tabla 6. Tipos de CM indicados. } \\
\hline Tipos de CM indicados & Número de pacientes \\
\hline $5 \%$ CBD- $0,2 \%$ THC & $252(71 \%)$ \\
$7,5 \%$ CBD- $0,2 \%$ THC & $103(29 \%)$ \\
Total & 355 \\
\hline
\end{tabular}

Tabla 8. Registro de efectos adversos durante el tratamiento.

\begin{tabular}{lc|}
\hline Efectos adversos & Número de pacientes \\
\hline Sin efectos colaterales & $183(51,5 \%)$ \\
Boca seca & $20(5,6 \%)$ \\
Mareos & $15(4,2 \%)$ \\
Somnolencia diurna & $22(6,2 \%)$ \\
Rash cutáneo & $1(0,3 \%)$ \\
No reconsultan & $46(13 \%)$ \\
Sin tratamiento & $35(9,9 \%)$ \\
Suspenden tratamiento & $15(4,2 \%)$ \\
Otros & $18(5,1 \%)$ \\
Total & $355(100 \%)$ \\
\hline
\end{tabular}

en $42 \%$ la mejoría fue leve a moderada y en $19 \%$ importante; $8 \%$ no notó cambios en sus síntomas (tabla 7).

4. Tolerancia y efectos adversos: en los pacientes tratados, 183 presentaron buena tolerancia al CM sin experimentar efectos adversos importantes, 75 refirieron efectos adversos de entidad leve (grado 1 de CTCAE), en grado de certeza posible y que revirtieron espontáneamente luego de algunos días (boca seca, mareos, dolor abdominal, constipación, somnolencia diurna, gastritis, hiperemia conjuntival, hipotensión, hipertensión) y un paciente experimentó un rash peribucal que requirió suspensión del tratamiento sin poner en peligro su vida (tabla 8).

\section{Discusión}

De la revisión bibliográfica surge que este es el primer estudio epidemiológico de evolución clínico terapéutica realizado en pacientes uruguayos en tratamiento con aceite de cannabis con alto contenido en CBD. Previamente se han publicado en el país algunas encuestas de divulgación sobre usos de $\mathrm{CM}^{(16)}$ que no recogen datos 


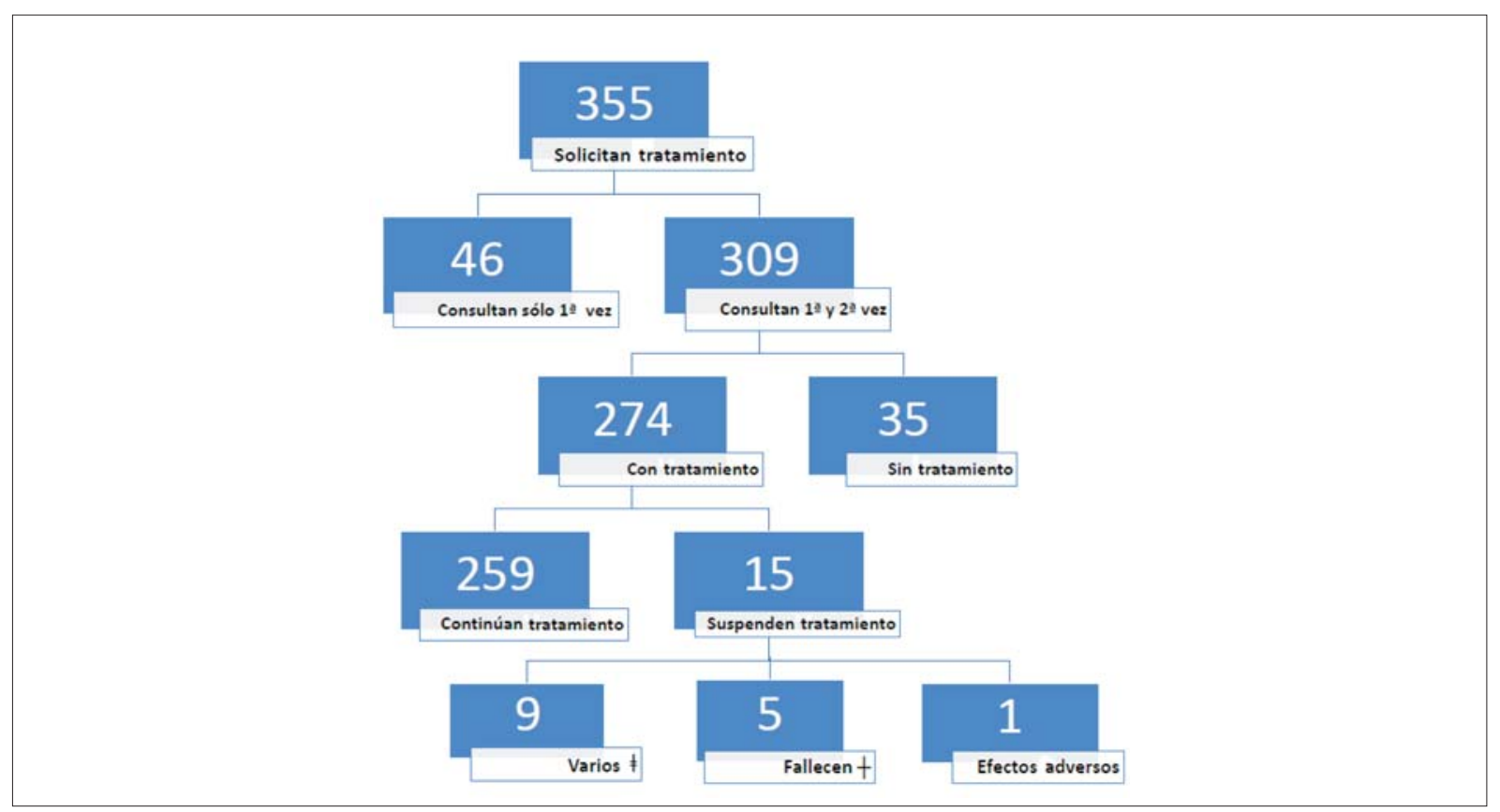

Figura 1. Diagrama de flujo que muestra la distribución de pacientes tras la consulta de medicina cannábica. (‡: costo elevado o problemas para realizar gestión; †: pacientes con cáncer en etapa terminal).

de tratamiento. Los resultados obtenidos muestran las características demográficas y patológicas de los pacientes, la elevada percepción del beneficio terapéutico observado con $\mathrm{CM}$ con alto contenido de CBD en diferentes contextos clínicos, la ausencia de efectos adversos severos, y las dificultades tanto económicas como de gestión que enfrentan quienes desean utilizarlo. La cohorte estudiada revela que la mayoría de los pacientes son mujeres y que predominan los mayores de 60 años, lo cual nos diferencia de otras poblaciones, como la norteamericana, con predominio masculino y edades de consulta más tempranas ${ }^{(17-19)}$. Este hecho puede relacionarse con la pirámide de población envejecida de nuestro país y la prevalencia femenina en las edades más avanzadas. Podría relacionarse también con el mayor poder adquisitivo de este grupo etario, reflejado en la pertenencia masiva a mutualistas (instituciones de atención médica colectiva) y seguros privados de salud con respecto a los servicios de atención públicos (hospitales del estado). La tasa elevada de escolarización terciaria $(45,6 \%)$ también nos diferencia de otros estudios epidemiológicos con $\mathrm{CM}$, como lo refieren los trabajos de Nunberg ${ }^{(17)}$ y O'Connell ${ }^{(20)}$, que muestran predominio de pacientes que solo finalizaron la educación secundaria. La escolarización terciaria apunta igualmente hacia una probable mejor situación económica (media a alta) y un nivel intelectual con capacidad para discernir y elegir consultar a profesionales médicos especializados en el tema y realizar gestiones con gastos pecuniarios que posibiliten el tratamiento. Nuestro estudio revela que las dificultades en las gestiones de obtención y el costo elevado del producto fueron las causas determinantes para no continuar su uso. Estos hechos nos cuestionan sobre el posicionamiento del CM dentro de las posibilidades terapéuticas. Al momento de la publicación de este trabajo, su utilización no tiene alcance universal porque las condiciones actuales de costos solo permiten el acceso a las personas con cierto poder adquisitivo dentro del espectro social. La falta de aprobación de fármacos cannábicos producidos a nivel nacional puede jugar un rol en este aspecto. Los prejuicios y la falta de formación especializada en el tema también contribuyen a la utilización informal de productos sin el soporte profesional adecuado. En cuanto a los síntomas y las patologías de consulta, estos apuntan mayoritariamente a la esfera neurológica psiquiátrica de tipo crónico y de grado severo, destacando la insuficiencia relativa de los tratamientos médicos convencionales frente a ésta. El dolor crónico refractario al tratamiento como consulta primaria es un hallazgo coincidente en trabajos previos $^{(21)}$, así como las expectativas en mejorar la calidad de vida. Respecto a los resultados terapéuticos, los aceites con alta proporción de CBD demostraron buena percepción de beneficios, observados en la mayoría de los pacientes y en todas las patologías de consulta. Debe tenerse presente que el efecto placebo puede formar parte de esta percepción, que sería más robusta si se mantiene en consultas posteriores. Este trabajo es un estudio pre- 
liminar que presenta limitaciones inherentes a los estudios observacionales: sesgos de selección, ausencia de seguimiento de varios pacientes que no retornaron a la consulta, pacientes que debieron suspender la medicación por falta de recursos para obtenerla. No son frecuentes los estudios observacionales con $\mathrm{CBD}$, exceptuando los realizados recientemente para la aprobación de Epidiolex ${ }^{\circledR}$ en epilepsia refractaria ${ }^{(22)}$. La mayoría de los ensayos clínicos y otros trabajos han utilizado proporciones iguales de CBD: THC (Nabiximols) ${ }^{(23)}$, THC sintético (Dronabinol) ${ }^{(24)} \mathrm{u}$ otros compuestos con THC como cannabinoide principal. Con respecto a efectos adversos y tolerancia, esta cohorte presentó efectos adversos leves que desaparecieron en los primeros días de uso o cumpliendo con la toma del aceite cercano a las comidas, datos concordantes con otros trabajos publica$\operatorname{dos}^{(25,26)}$. Solo una paciente con rash peribucal requirió suspensión del tratamiento. Ninguno de los pacientes tuvo riesgo vital por dichos eventos.

\section{Conclusiones}

Uruguay es un país pionero en la regulación y el uso de cannabis tanto en forma recreativa como medicinal. Los resultados de nuestro estudio reflejan la experiencia preliminar de los beneficios observados y tolerancia, con escasos y leves efectos adversos de CM con alto contenido de CBD en múltiples indicaciones. Esos resultados constituyen evidencia que respalda clínicamente a las propuestas para la disminución de costos y acceso universal al CM siguiendo pautas médicas.

De la población atendida, se destaca que la mayoría fue de sexo femenino, mayor de 60 años, con patologías neurológicas y dolor crónico por patología osteoarticular refractario a la terapéutica convencional, con nivel terciario de estudios, usuarios de salud privada, predominando la expectativa de mejorar su calidad de vida. Respondieron positivamente al tratamiento $60,6 \%$ de los casos, en $18,7 \%$ con mejoría importante y leve a moderada en $41,7 \%$.

\section{Abstract}

Introduction: Cannabis Sativa L. products are known to be therapeutically effective since ancient times, despite its pharmacological typification was made in the mid- $20^{\text {th }}$ century. Its use was recently regulated in Uruguay.

Objective: to analyse a preliminary clinical-therapeutic experience with $\mathrm{MC}$ with high content of cannabidiol (CBD).

Method: epidemiological, observational and retrospective study of a 355 patient cohort who spontaneously consulted to learn about $\mathrm{CM}$ at a private clinic, between August 2016 and December 2017.
Demographic data, medical records, expectation and previous experience with cannabis were collected in the first interview. In most cases, cannabis with high content of CBD was prescribed $(5,25 \% \mathrm{CBD}$ and $0,2 \%$ THC).

In subsequent consultations, access to access to $\mathrm{MC}$ was invesrtigated and both response to it and adverse effects were studied by means of analogue scales. The study used descriptive statistics.

Results: in the cohort studied, women with an average age of 67 years old and university studies prevailed. The following conditions motivated consultations: neurological (38\%), rheumatic or bone degenerative diseases (37\%), neoplasms (13\%), psychiatric diseases (4\%) and miscelánea (8\%). In most cases $(60,6 \%)$ patients stated symptoms improved and only $16,3 \%$ of the populaiton studied presented mild adverse effects. High costs and difficulties in accessing $\mathrm{MC}$ were the reasons for not starting or abandoning treatment.

Conclusions: our preliminary study reflects the positive therapeutic response and non significant adverse effects to MC with high content of CBD. $60,6 \%$ of patients treated referred improvement in their symptoms. Decisive factors for a successful treatment point at the need to make access to MC easier by improving management to obtain it and reducing costs for a greater accessibility.

\section{Resumo}

Introdução: A eficácia terapêutica dos produtos de Cannabis Sativa L. é conhecida desde a antiguidade, embora sua tipificação farmacológica tenha sido feita a meados do século XX. Seu uso no Uruguai foi regularizado recentemente.

Objetivo: Analisar uma experiencia clínico-terapêutica preliminar com $\mathrm{CM}$ com alto teor de Cannabidiol (CBD).

Métodos: Estudo epidemiológico observacional e retrospectivo de uma coorte de 355 pacientes que consultaram espontaneamente sobre $\mathrm{CM}$ em uma clínica privada no período agosto de 2016 - dezembro de 2017. Durante a primeira entrevista foram coletados dados demográficos, clínicos, farmacológicos, expectativas e experiencia previa com cannabis. Na maioria dos casos indicou-se cannabis com alto teor em CBD $(5,25 \%$ de CBD e $0,2 \%$ de THC). Nas consultas seguintes, coletou-se informação sobre o acesso a $\mathrm{CM}$ e realizou-se uma avaliação da resposta e dos efeitos adversos utilizando escalas analógicas. Foram utilizados métodos de estadística descritiva.

Resultados: Na coorte estudada predominaram mulheres com idade média de 67 anos, de nível educativo terciário. As razõoes de consulta foram patologias 
neurológicas (38\%), enfermidades reumáticas ou artro-degenerativas $(37 \%)$, neoplasias $(13 \%)$, doenças psiquiátricas (4\%) e outras (8\%). A maioria dos casos $(60.6 \%)$ informou melhoria dos sintomas e somente $16.3 \%$ da população estudada apresentou efeitos adversos leves. As causas para não iniciar ou abandonar o tratamento foram os altos custos e as dificuldades para a obtenção da CM.

Conclusões: Este estudo preliminar mostra uma resposta terapêutica positiva e sem efeitos adversos significativos ao CM com alto teor de CBD. $60.6 \%$ dos pacientes tratados informou melhoria de sintomas. Os fatores decisivos para um tratamento exitoso mostram a necessidade de facilitar o acesso a CM melhorando a gestão para sua obtenção e diminuindo os custos para aumentar o acesso.

\section{Bibliografía}

1. Russo EB. History of cannabis and its preparations in saga, science, and sobriquet. Chem Biodivers 2007; 4(8):1614-48.

2. Hanuš L. How and why Cannabis became such famous medicine. En: Coloquio "El cannabis y los cannabinoids en la medicina" (27 de octubre de 2017). Montevideo: Sociedad Uruguaya de Endocannabinología, 2017.

3. Lu HC, Mackie K. An introduction to the endogenous cannabinoid system. Biol Psychiatry 2016; 79(7):516-25.

4. Elsohly MA, Gul W. Constituents of cannabis sativa. En: Pertwee RG, ed. Handbook of cannabis. Oxford, UK: Oxford University, 2014:4-19.

5. Pazos Rodríguez MR, Grandes Moreno P. Cannabinoides y sistema endocannabinoide. En: Ramos Atance JA, coord. Efectos terapéuticos de los cannabinoides. Madrid: Universidad Complutense de Madrid, 2017:7-23. Disponible en: http://www.seic.es/wp-content/uploads/2013/10/EFECTOSTERAP\%C3\%89UTICOS-DE-LOS-CANNABINOIDES.p df. [Consulta: 24 marzo 2018].

6. Elsohly MA, Slade D. Chemical constituents of marijuana: the complex mixture of natural cannabinoids. Life Sci 2005; 78(5):539-48

7. Bewley-Taylor D, Blickman T, Jelsma M. Auge y caída de la prohibición del cannabis. Ámsterdam: Trasnational Institute, 2014. Disponible en:https://www.tni.org/files/download/auge-y-caidaweb.pdf [Consulta: 20 marzo 2018].

8. Friedrich-Ebert-Stiftung Uruguay. Estrategia para la evaluación de resultados y monitoreo de implementación de la Ley 19.172: propuesta. Montevideo: FESUR, 2014. Disponible en: http://monitorcannabis.uy/wp-content/uploads /2016/03/Estrategia-EYM-FESUR-11-2015-V6.pdf [Consulta: 27 marzo 2018]

9. Uruguay. Ley 19.172. Marihuana y sus derivados: control y regulación del estado de importación, producción, adquisición, almacenamiento, comercialización y distribución. Montevideo, 20 de diciembre de 2013.
10. Uruguay. Decreto $N^{\circ} 46 / 015$. Reglamentación de la Ley 19.172 relativa a la regulación y control del cannabis. Montevideo, 4 de febrero de 2015.

11. Uruguay. Decreto $N^{\circ} 298 / 2017$. Autorización de "venta bajo receta profesional" para las especialidades farmacéuticas con cannabidiol como principio activo. Montevideo, 16 de octubre de 2017

12. Schachter M. Unwanted effects and adverse drug reactions. En: Bennett PN, Brown MJ, Sharma P, eds. Clinical pharmacology. 11 ed. Edinburgh: Elsevier, 2012:110-21.

13. National Cancer Institute. National Institutes of Health Department of Health and Human Services. Common terminology criteria for adverse events (CTCAE) version 5.0. Bethesda, MD: NCI, 2017. Disponible en:https://ctep.cancer.gov/protocolDevelopment/electronic_applications/docs/CTCAE_v5_Quick_Reference_8.5x11.pdf. [Consulta: 20 marzo 2018].

14. A fifth amendment for the Declaration of Helsinki. Lancet 2000; 356(9236):1123.

15. Asociación Médica Mundial. Declaración de Taipei: investigación sobre las bases de datos, datos masivos y biobancos. Taipei: AMM, 2016.

16. Robaina G, Peyraube R. Cannabis medicinal: la revolución silenciosa. Montevideo: Monitor Cannabis Uruguay, 2018. Disponible en:http://monitorcannabis.uy/cannabis-medicinal-la-revolucion-silenciosa/ [Consulta: 25 marzo 2018]

17. Nunberg H, Kilmer B, Pacula RL, Burgdorf J. An analysis of applicants presenting to a medical marijuana specialty practice in California. J Drug Policy Anal 2011; 4(1):1-14. Disponible en: https://www.ncbi.nlm.nih.gov/pmc/articles/PMC3673028/ [Consulta: 27 marzo 2018].

18. Reinarman C, Nunberg H, Lanthier F, Heddleston T. Who are medical marijuana patients? Population characteristics from nine California assessment clinics. J Psychoactive Drugs 2011; 43(2):128-35.

19. Fairman BJ. Trends in registered medical marijuana participation across 13 US states and District of Columbia. Drug Alcohol Depend 2016; 159:72-9.

20. O'Connell TJ, Bou-Matar CB. Long term marijuana users seeking medical cannabis in California (2001-2007): demographics, social characteristics, patterns of cannabis and other drug use of 4117 applicants. Harm Reduct J 2007; 4:16.

21. Abuhasira R, Schleider LB, Mechoulam R, Novack V. Epidemiological characteristics, safety and efficacy of medical cannabis in the elderly. Eur J Intern Med 2018; 49:44-50.

22. U.S. Food and Drug Administration. FDA approves first drug comprised of an active ingredient derived from marijuana to treat rare, severe forms of epilepsy. Silver Spring, MD: FDA, 2018. Disponible en:https://www.fda.gov/NewsEvents/Newsroom/PressAnnouncements/ucm611046.htm [Consulta: 25 marzo 2018]

23. Flachenecker P, Henze T, Zettl UK. Nabiximols (THC/CBD oromucosal spray, Sativex ${ }^{\circledR}$ ) in clinical practice - results of a multicenter, non-interventional study (MOVE 
2) in patients with multiple sclerosis spasticity. Eur Neurol 2014; 71(5-6):271-9.

24. Badowski ME, Yanful PK. Dronabinol oral solution in the management of anorexia and weight loss in AIDS and cancer. Ther Clin Risk Manag 2018; 14:643-51.

25. Bellnier T, Brown GW, Ortega TR. Preliminary evaluation of the efficacy, safety, and costs associated with the treatment of chronic pain with medical cannabis. Ment Health Clin 2018; 8(3):110-5.

26. Iffland K, Grotenhermen F. An update on safety and side effects of cannabidiol: a review of clinical data and relevant animal studies. Cannabis Cannabinoid Res 2017; 2(1):139-54.

\section{Contribución de autores}

Julia Galzerano, https://orcid.org/0000-0001-7521-2145. Concepción, diseño, ejecución, análisis, redacción y revisión crítica.

Cecilia Orellano, https://orcid.org/0000-0002-9416-3833. Concepción, diseño, ejecución, análisis, redacción y revisión crítica.

Daniela Ríos, https://orcid.org/0000-0002-3635-6987. Ejecución y análisis.

Ana Coitiño, https://orcid.org/0000-0002-4732-0752. Ejecución y análisis.

Mariano Velázquez, https://orcid.org/0000-0002-6850-6310. Ejecución y análisis. 